https://doi.org/10.21278/TOF.444013820

ISSN 1333-1124

eISSN 1849-1391

\title{
BUCKLING AND VIBRATION OF A STEPPED PLATE
}

\begin{abstract}
Summary
This study analyses the elastic stability and free vibration of a simply supported stepped plate under combined loading conditions defined by the parameter $\alpha$. Mathematical identification of these phenomena has been made using Levy's method as implemented in the conditions of equivalent fictitious load. The buckling coefficient $k$ and the frequency parameter $\lambda$ of the stepped plate were verified according to literature sources. Influential parameters of stability and the free vibration of the stepped plate under combined load were identified. It has been concluded that the buckling coefficient primarily depends on the relative thickness $\Delta t$, while the frequency parameter $\lambda$ was significantly affected by the position of discontinuity $\Delta b$. Pure bending $(\alpha=2)$ induces several buckling modes for the same plate geometry with respect to uniform compression $(\alpha=0)$, thus creating a considerable technological stability reserve, particularly at higher discontinuities. Formulation of the frequency parameter enables us to choose the optimum geometry with minimal susceptibility to the appearance of free vibration in the plate.
\end{abstract}

Key words: $\quad$ stability, vibration, stepped plate, buckling coefficient, frequency parameter.

\section{Introduction}

Plates of stepwise variable thickness are often present as elements in optimally designed structures with high performance requirements (e.g. aircraft elements). In the last ten years numerous publications using different methods have provided significant results in the field of buckling and vibration of thin plates [1-5]. The largest number of studies on the stability and vibration of plates of stepwise variable thickness refer to the cases of uniform compressive loading under various boundary conditions [6-8]. These studies based their research on the classical theory of thin plates (Kirchhof's method) and resolve it using Levy's function; theoretical foundations of these studies are provided in [9]. The methodology based on this approach allows for the implementation of Kirchhof's method when analysing the stability and vibration of plates with one of two discontinuities [10]. Linearly varying load is often present in technical practice as a result of bending moment; in some studies, this effect was analysed only for the case of uniform plates [11-12]. A significant contribution in this regard is offered by studies [13-14], where the elastic stability of a uniform longitudinally stiffened plate was studied using the Galerkin method. 


\begin{tabular}{|ll|}
\hline$a$ & Nomenclature \\
$b$ & Plate length \\
$t_{i}$ & Plate width \\
$b_{i}$ & Whickness of the $i$-th plate segment \\
$i$ & Number of segments in the stepped plate of variable thickness $(i=2)$ \\
$D_{i}$ & Stiffness of the $i$-th plate segment $\left\{E t_{i}^{3} /\left[12\left(1-v^{2}\right)\right]\right\}$ \\
$E$ & Elasticity modulus (for steel $\left.21000 \mathrm{kN} / \mathrm{cm}^{2}\right)$ \\
$v$ & Poisson's ratio (for steel 0.3$)$ \\
$x, y$ & Coordinates of the Cartesian system \\
$w_{i}(x, y, t)$ & Dynamic traverse plate displacement $(\mathrm{deflection})$ \\
$W_{i}(x, y)$ & Static traverse plate displacement $(\mathrm{deflection})$ \\
$\sigma_{x}, \sigma_{v}, \sigma_{x v}$ & Components of plane stresses \\
$\rho$ & Density of the plate material $\left(\right.$ for steel $\left.7850 \mathrm{~kg} / \mathrm{m}^{3}\right)$ \\
$\ddot{w}$, & Acceleration of traverse plate displacement \\
$\omega_{n}$ & Natural frequency of plate vibration \\
$\Delta t$ & Relative plate thickness $\left(t_{1} / t_{2}\right)$ \\
$\Delta b$ & Relative plate width $\left(b_{1} / b\right)$ \\
$m, n$ & Number of buckling and vibration modes \\
$k$ & Elastic buckling coefficient $\left\{N_{c r} b^{2} /\left(\pi^{2} D_{2}\right)\right\}$ \\
$\lambda$ & Frequency parameter $\left\{\left(\omega_{n} a^{2} / \pi^{2}\right)\left[\rho t_{2} / D_{2}\right]^{1 / 2}\right\}$ \\
\hline
\end{tabular}

Numerical approaches such as the finite difference method (FDM) are suitable for resolving the issue of stability in arbitrary boundary conditions, where the accuracy of results depends on the number of nodal points generated [15]. The finite strip method (FSM) provides a wide range of possibilities for studying the stability and the eigenfrequencies of oscillations of rectangular plates in complex supporting conditions [16-17]. As a recent approach, the differential square method (DQM) is also used in the analysis of buckling and free vibration of plates; its implementation enables the analysis of inclined plates [18]. The approximate procedure, discussed in [19], which is based on a modified form of buckling mode is a significant contribution to this field. Effects of the higher-order shear stress on Levy-type solutions for dealing with the plate buckling is presented in [20]; this is important for the analysis of structural elements with high shear stresses (e.g. in the girder support zone). An analogy can be drawn between the buckling and vibration phenomena in terms of the plate behaviour, although the causes of critical state are indicated differently. The dynamic stability of isotropic or orthotropic plates is studied in [21] based on the static component and using the buckling coefficient, while the dynamic susceptibility of stepped plates is analysed in [22] based on the effect of free vibration. A simplified dynamic analysis of stepped plates is presented in [23]. Structural analysis of thin plates with the finite element method is given in [24-25]. The new finite element formulation has been successfully applied to vibration analysis of thin plates [26].In this sense, it is important for the frequency of dynamic change in the external load not to overlap with the plate frequencies. In this respect, the present study aims to complement the lack of data on the critical buckling force and the frequency parameter of stepwise variable thickness plates subjected to uniform and linearly varying loads. 


\section{Mathematical modelling}

The most general interpretation of buckling and free (harmonic) vibration phenomena in the field of elastic behaviour of an isotropic plate which is loaded only by external forces is expressed through the differential equation of the following form:

$$
D_{i}\left(\frac{\partial^{4} w_{i}}{\partial x^{4}}+2 \frac{\partial^{4} w_{i}}{\partial x^{2} y^{2}}+\frac{\partial^{4} w_{i}}{\partial y^{4}}\right)+\sigma_{x} t_{i} \frac{\partial^{2} w_{i}}{\partial x^{2}}+\sigma_{y} t_{i} \frac{\partial^{2} w_{i}}{\partial y^{2}}+\sigma_{x y} t_{i} \frac{\partial^{2} w_{i}}{\partial x \partial y}-\rho t_{i} \ddot{w}_{i}=0
$$

To solve the partial differential equation (1), it is necessary to separate the variables that are related to $x$ and $y$ coordinates and time $t$ so that the deflection function $w_{i}$ is assumed in the form of the product of two independent functions:

$$
w_{i}(x, y, t)=W_{i}(x, y) \sin \left(\omega_{n} t\right)
$$

The research in this study is focused on the stability of the box girder vertical plate ribs (Fig. 1). The prevailing stress of the box girder comes from the bending moment (in the vertical plane) and the localized effect of the load. The bending moment of the vertical plates $\left(M_{r}\right)$ is proportional to the axial moment of their surface areas and the overall bending moment of the girder. Global stresses in terms of optimum design require an increase in the girder height, and consequently that of the rib; this result in increased slenderness and a potential loss of stability due to a greater bending moment [27].

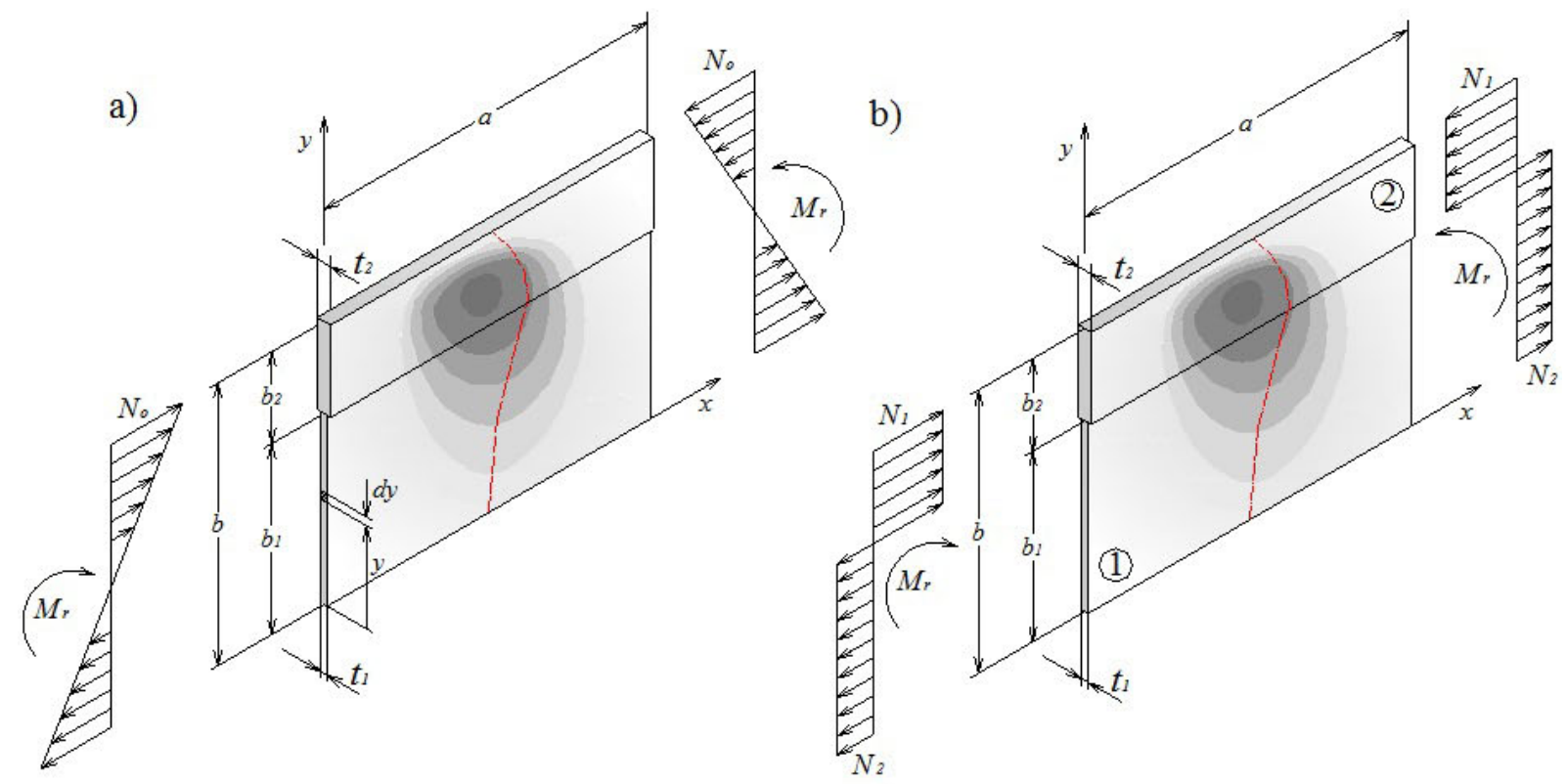

Fig. 1 A plate loaded: a) by linearly varying load from the moment $M_{r}$ (actual load) and b) by equivalent constant forces $N_{1}$ and $N_{2}$ (fictitious load)

The main problem regarding the implementation of equation (1) for vertical girder plates is not related only to the geometry (stepwise variable thickness), but also to the conditions of supporting (boundary conditions), as well as the characteristics of loading. Studies dealing with the issue of elastic stability of plates subjected to a linearly varying load use numerical methods. The present study discusses a procedure of equivalent fictitious load that offers the possibility of implementation in equation (1) through a constant value. The idea behind this approach is based on substituting the actual load (Fig. 1a) with a fictitious load (Fig. 1b) that has an equivalent effect on the plate behaviour. Values of fictitious forces $N_{1}$ and $N_{2}$ are given in Appendix A. 
By substituting (2) into (1) and bearing in mind the previous analysis we have that:

$$
\left(\frac{\partial^{4} W_{i}}{\partial x^{4}}+2 \frac{\partial^{4} W_{i}}{\partial x^{2} y^{2}}+\frac{\partial^{4} W_{i}}{\partial y^{4}}\right)+\frac{\sigma_{x} t_{i}}{D_{i}} \frac{\partial^{2} W_{i}}{\partial x^{2}}+\frac{\rho t_{i} \omega^{2}}{D_{i}} W_{i}=0
$$

If the plate is simply supported at two opposite edges $x=0$ and $x=a$, then the function along the $x$-axis can be assumed in the form of a harmonic function, i.e.

$$
W_{i}(x, y)=\sum_{m=1}^{\infty} X_{m, i}(x) Y_{m, i}(y)=\sum_{m=1}^{\infty} \sin \left(\frac{m \pi x}{a}\right) Y_{m, i}(y)
$$

Substituting (4) into (3) leads to an ordinary linear differential equation of the fourth order with the $y$ variable, which has the following form:

$$
Y_{m, i}^{\prime \prime \prime \prime}-\left[2\left(\frac{m \pi}{a}\right)^{2}\right] Y_{m, i}^{\prime \prime}+\left[\left(\frac{m \pi}{a}\right)^{4}\left(1-\frac{\sigma_{x, i} t_{i} a^{2}}{m^{2} \pi^{2} D_{i}}-\frac{\omega_{n}^{2} a^{4} \rho t_{i}}{m^{4} \pi^{4} D_{i}}\right)\right] Y_{m, i}=0
$$

or in a shorter form:

$$
Y_{m, i}^{\prime \prime \prime}-\left(2 \varphi \varepsilon_{m}\right) Y_{m, i}^{\prime \prime}+\left(\varphi^{4} \delta_{m}\right) Y_{m, i}=0,
$$

where $\varphi, \varepsilon_{m}$ and $\delta_{m}$ are given in Appendix B.

The general solution to equation (6) has the following form [28]:

$$
[\chi]_{i}=e^{[A] y}=[F(y)][F(0)]^{-1}
$$

where, with $[\chi]_{i}$, the form of the solution for $Y_{m, i}$ is assumed.

The matrix $[F(y)]$ is formed by the functions of eigenvalues $r_{q}$ and eigenvectors $v_{q}$.

$$
F_{q}(y)=e^{r_{q} y} v_{q}, \quad q=1,2, \ldots, 4 .
$$

Eigenvalues of matrix $[A]$ define the roots of the characteristic equation (6):

$$
r_{1234}= \pm \varphi \sqrt{\varepsilon_{m} \pm \sqrt{\varepsilon_{m}^{2}-\delta_{m}}}
$$

There are three cases or forms of function (4), bearing in mind that in all cases $\left(\varphi, \varepsilon_{m}\right)>0$.

The first case: $\varepsilon_{m}{ }^{2}<\delta_{m}$

$$
\begin{aligned}
& W_{i}(x, y)=\sum_{m=1}^{\infty}\left[\begin{array}{l}
A_{m} \sinh \left(\alpha_{m} y\right) \sin \left(\beta_{m} y\right)+B_{m} \sinh \left(\alpha_{m} y\right) \cos \left(\beta_{m} y\right)+ \\
C_{m} \cosh \left(\alpha_{m} y\right) \sin \left(\beta_{m} y\right)+D_{m} \cosh \left(\alpha_{m} y\right) \cos \left(\beta_{m} y\right)
\end{array}\right] \sin \left(\frac{m \pi x}{a}\right) \\
& \alpha_{m}=\varphi \sqrt{\frac{\sqrt{\delta_{m}}+\varepsilon_{m}}{2}} \text { and } \beta_{m}=\varphi \sqrt{\frac{\sqrt{\delta_{m}}-\varepsilon_{m}}{2}}
\end{aligned}
$$

The second case: $\varepsilon_{m}{ }^{2}>\delta_{m}, \delta_{m}<0$

$$
\begin{aligned}
& W_{i}(x, y)=\sum_{m=1}^{\infty}\left[\begin{array}{l}
A_{m} \sinh \left(\alpha_{m} y\right)+B_{m} \cosh \left(\alpha_{m} y\right)+ \\
C_{m} \sin \left(\beta_{m} y\right)+D_{m} \cos \left(\beta_{m} y\right)
\end{array}\right] \sin \left(\frac{m \pi x}{a}\right) \\
& \alpha_{m}=\varphi \sqrt{\sqrt{\varepsilon_{m}^{2}-\delta_{m}}+\varepsilon_{m}} \text { and } \beta_{m}=\varphi \sqrt{\sqrt{\varepsilon_{m}^{2}-\delta_{m}}-\varepsilon_{m}}
\end{aligned}
$$


The third case: $\varepsilon_{m}{ }^{2}>\delta_{m}, \delta_{m}>0$

$$
\begin{aligned}
& W_{i}(x, y)=\sum_{m=1}^{\infty}\left[\begin{array}{l}
A_{m} \sinh \left(\alpha_{m} y\right)+B_{m} \cosh \left(\alpha_{m} y\right)+ \\
C_{m} \sinh \left(\beta_{m} y\right)+D_{m} \cosh \left(\beta_{m} y\right)
\end{array}\right] \sin \left(\frac{m \pi x}{a}\right) \\
& \alpha_{m}=\varphi \sqrt{\varepsilon_{m}+\sqrt{\varepsilon_{m}^{2}-\delta_{m}}} \text { and } \alpha_{m}=\varphi \sqrt{\varepsilon_{m}-\sqrt{\varepsilon_{m}^{2}-\delta_{m}}}
\end{aligned}
$$

The parameters $A_{m}, B_{m}, C_{m}$, and $D_{m}$ of the mode $m$ are determined according to the given boundary conditions of the plate support and the compatibility conditions at the plates connection.

Segment 1:

$$
\begin{array}{ll}
W_{l}=0, \quad \text { for } y=0 & W_{2}=0, \quad \text { for } y=b \\
\frac{\partial^{2} W_{1}}{\partial y^{2}}=0, \text { for } y=0 & \frac{\partial^{2} W_{2}}{\partial y^{2}}=0, \text { for } y=b
\end{array}
$$

Segments 1 and 2 are functionally connected into an inseparable unit along the line $y=b_{1}$, which ensures the conditions of plate displacement continuity and stress compatibility in the zone of discontinuity. Functions of deflection, inclination, bending moments and transverse forces along the connection line between the two segments of the observed plate system must be all continuous functions, which is mathematically formulated as follows:

$$
\begin{aligned}
& W_{1}=W_{2} \\
& \left.\left(\frac{\partial W_{1}}{\partial y}\right)\right|_{y=b_{1}}=\left.\left(\frac{\partial W_{2}}{\partial y}\right)\right|_{y=b_{1}}=\left.D_{2}\left(\frac{\partial^{2} W_{2}}{\partial y^{2}}+v \frac{\partial^{2} W_{2}}{\partial x^{2}}\right)\right|_{y=b_{1}} \\
& \left.D_{1}\left(\frac{\partial^{2} W_{1}}{\partial y^{2}}+v \frac{\partial^{2} W_{1}}{\partial x^{2}}\right)\right|_{y=b_{1}}=\left.D_{2}\left(\frac{\partial^{3} W_{2}}{\partial y^{3}}+(2-v) \frac{\partial^{2} W_{2}}{\partial x^{2} \partial y}\right)\right|_{y=b_{1}}
\end{aligned}
$$

The introduction of an equivalent fictitious load clearly indicates that the element 1 is subjected to tension and the element 2 to compression. The minimum values of the elastic buckling coefficient $k$ and free vibrations for the plate $\lambda$ need to be determined from the following homogeneous systems of algebraic equations:

$$
\left[K_{s}\right]_{8 \times 8}\{C\}=\{0\}
$$

$\left[K_{s}\right]_{8 \times 8}$ - matrix of the boundary and compatibility conditions of the plate formed based on (16)-(21);

$\{\mathrm{C}\}-$ matrix of unknown coefficients $\left(=\left\{A_{m, 1} B_{m, 1} C_{m, 1} D_{m, 1} A_{m, 2} B_{m, 2} C_{m, 2} D_{m, 2}\right\}^{\mathrm{T}}\right)$. 


\section{Elastic buckling of a stepped plate}

The developed mathematical model discussed in Section 2 provides an exact solution when analysing the issue of elastic buckling and free vibration of stepped plates with stepwise in-plane load. The functional dependence of the elastic buckling coefficient $k$ on the geometric parameters of the stepped plate $(\Delta t, \Delta b$, and $a / b)$ is given in Fig. 2. Increasing the relative plate thickness leads to an increase in the value of the parameter $k$, while the effect of the first buckling mode ranges from the value of $a / b=1.4$ for $\Delta t=1.0$ to the value of $a / b=1.6$ for $\Delta t=1.4$. Larger plate discontinuities indicate more significant changes to the buckling coefficient $k$ in the interval of $a / b=1-2$, indicating a considerable technological reserve in the plate with respect to the minimum values. The parameter $k$ has a linear trend of increase with the decreasing relative plate width $\Delta b$. With the increasing $a / b$, values of the function $k$ display a clear tendency towards an asymptotic value; thus, for $a / b>2$, the minimum value $k_{\min }$ is being assumed.
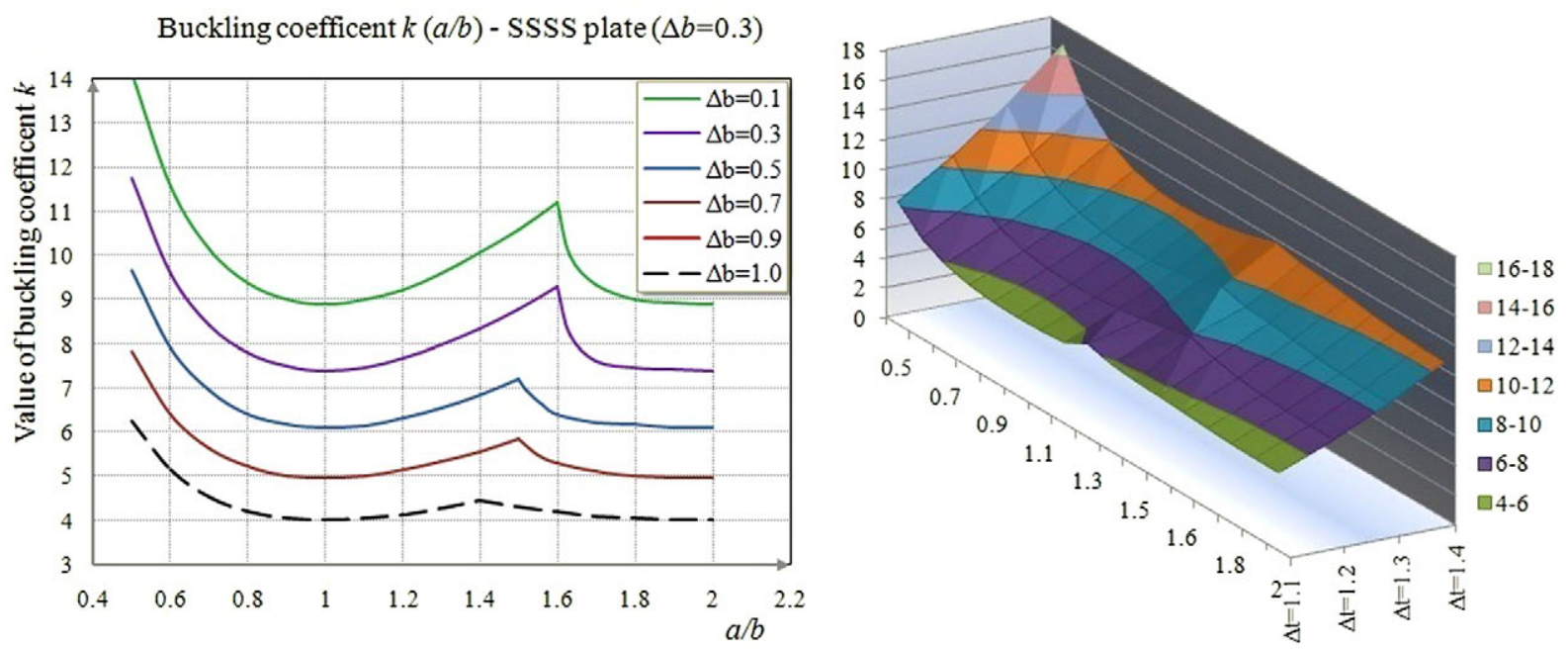

Buckling coefficent $k(a / b)$ - SSSS plate $(\Delta t=1.2)$
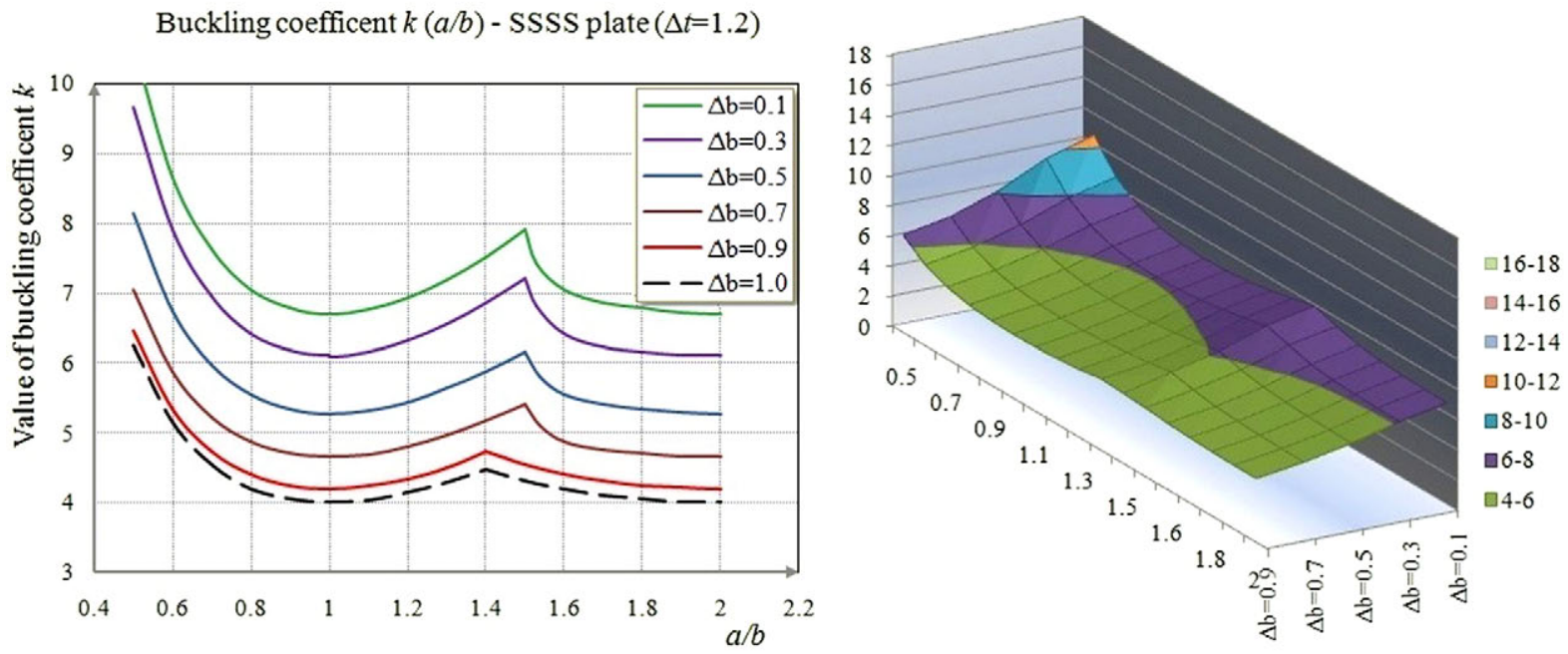

Fig. 2 Dependence of the buckling coefficient $k$ on the plate geometry (for $\alpha=0$ )

Exceptions from this rule are large discontinuities $(\Delta t>2)$; in that case, it is reasonable to analyse the third or possibly higher buckling modes $(m \geq 3)$. The present analysis clearly indicates the necessity of using plates of stepwise variable thickness regardless of the fact that they are subjected to uniform pressure. 


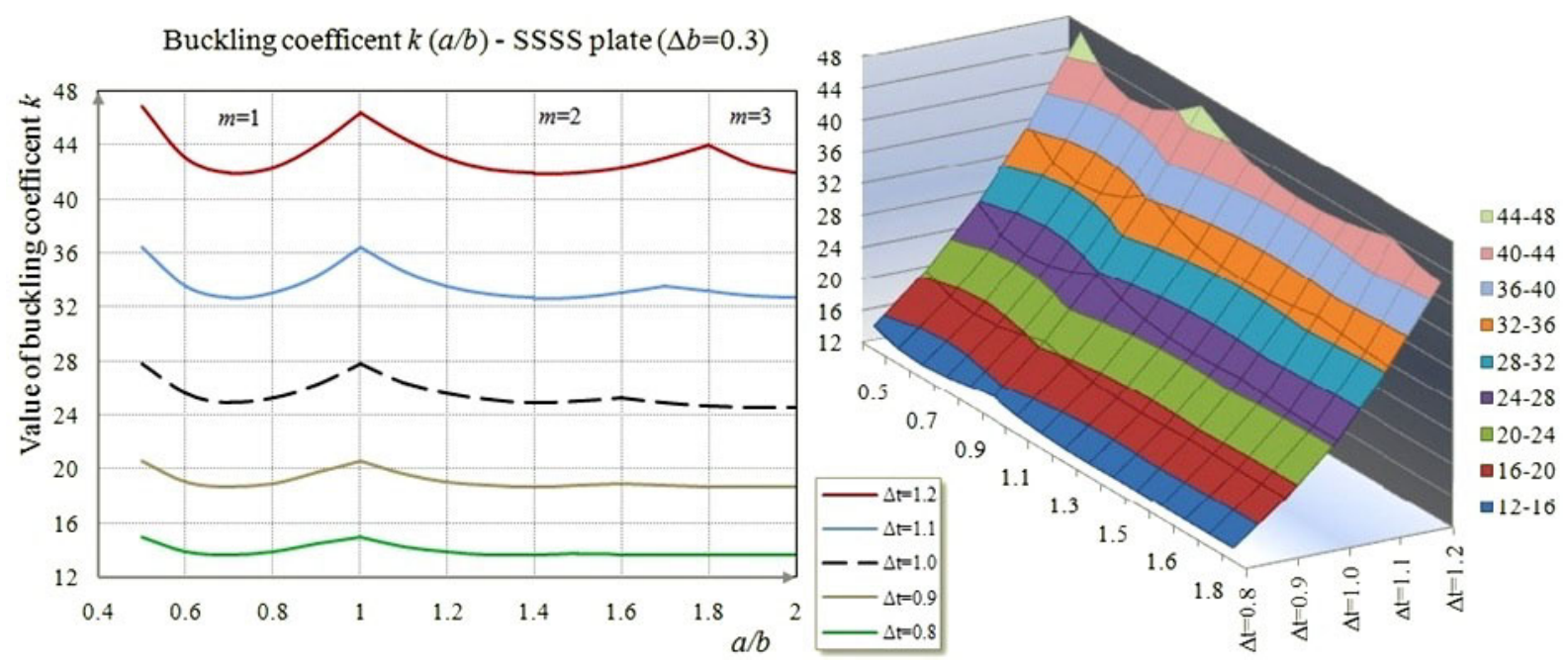

Fig. 3 Distribution of the buckling coefficient $k$ for $\alpha=2$

The analysis is performed only for fictitious load, Fig. 1b. For this load case $(\alpha=2)$, it is necessary to analyse the first three modes, while for larger discontinuities, higher buckling modes have to be analysed as well (Fig. 3). Other rules are as in the previous case; numerical values for the characteristic parameters of the stepped plate are given in Table 1. A value of $t_{1} / t_{2}=1.0$ is consistent with [8], which is given for the second approximation, with an average deviation of $3.8 \%$.

Table 1 Values of buckling coefficient $k$ for the first three modes $(\alpha=2)$

\begin{tabular}{cccccccccc}
\hline \multirow{2}{*}{$b_{2} / b$} & $t_{1} / t_{2}$ & \multicolumn{10}{c}{$a / b$} \\
\cline { 3 - 10 } & & 0.5 & 0.8 & 1.0 & 1.2 & 1.4 & 1.6 & 1.8 & 2.0 \\
\hline \multirow{3}{*}{0.3} & 0.8 & 14.992 & 13.876 & 14.922 & 13.870 & 13.631 & 13.705 & 13.640 & 13.630 \\
& 0.9 & 20.633 & 18.968 & 20.633 & 19.082 & 18.681 & 18.968 & 18.732 & 18.681 \\
& 1.0 & 27.758 & 25.322 & 27.760 & 25.586 & 24.984 & 25.322 & 24.708 & 24.608 \\
& 1.1 & 36.453 & 33.080 & 36.453 & 33.526 & 32.679 & 33.080 & 33.209 & 32.679 \\
& 1.2 & 46.877 & 42.383 & 46.377 & 43.046 & 41.906 & 42.383 & 43.964 & 41.906 \\
\hline \multirow{3}{*}{0.5} & 0.8 & 16.702 & 15.361 & 14.922 & 18.918 & 15.179 & 15.365 & 15.297 & 15.171 \\
& 0.9 & 21.618 & 19.792 & 20.633 & 19.991 & 19.539 & 19.792 & 19.543 & 19.530 \\
& 1.1 & 35.257 & 32.076 & 36.453 & 32.421 & 31.635 & 32.076 & 33.352 & 31.632 \\
& 1.2 & 44.251 & 40.176 & 46.377 & 40.619 & 39.611 & 40.176 & 41.811 & 39.611 \\
\hline
\end{tabular}

\section{Free vibration of a stepped plate}

The eigenfrequency of a plate with one discontinuity (two elements of different thickness) is defined by the frequency parameter $\lambda$. As indicated by the discussed mathematical model, there is an analogy between the buckling coefficient $k$ and the frequency parameter $\lambda$ in terms of loss of the plate stability. The elastic buckling coefficient $k$ is the basis for defining the critical in-plane stress that leads to the buckling of the plate as a result of external compression loading. The frequency parameter $\lambda$ enables us to determine the eigenfrequencies of the plate vibration, which is crucial in the analysis of the plate dynamic behaviour. Harmonic transverse displacement, i.e. deflection of the plate whose excitation (forcing) frequency of vibration $\omega$ corresponds to its own frequency $\omega_{n}$, or is very close to it, leads to the resonant behaviour or critical condition. 
It is important to note that the loss of plate stability in the case when $\omega \approx \omega_{n}$ can occur at much lower intensity of external load than in the case of static action. The eigenfrequency of vibration of plates of stepwise variable thickness $\omega_{n}$ is defined according to convention as

$$
\omega_{n}=\lambda\left(\frac{\pi}{a}\right)^{2} \sqrt{\frac{D_{2}}{\rho t_{2}}}
$$

The frequency parameter $\lambda$ is an exclusively geometric property which is functionally dependent on the length, width, and thickness of the element of which the plate of stepwise variable thickness is formed. The frequency parameter $\lambda$, depending on the relative thickness $\left(t_{1} / t_{2}\right)$, slenderness $(a / b)$, and the relative plate width $\left(b_{1} / b\right)$ for the first and second modes of vibration is formulated from the following expressions, respectively:

$$
\begin{aligned}
& \lambda_{1}\left(\frac{t_{1}}{t_{2}}, \frac{a}{b}, \frac{b_{1}}{b}\right)=\sqrt{\frac{B_{1}}{C}} \\
& \lambda_{2}\left(\frac{t_{1}}{t_{2}}, \frac{a}{b}, \frac{b_{1}}{b}\right)=\sqrt{\frac{B_{1}+B_{2}+\sqrt{\left(B_{1}+B_{2}\right)^{2}-4 B_{1} B_{2}}}{2 C}}
\end{aligned}
$$

Expressions for parameters $B_{1}, B_{2}$, and $C$ are given in Appendix C.

The three-dimensional functions of the frequency parameter for the first and second modes of plate vibration are provided in Fig. 4 and Fig. 5.
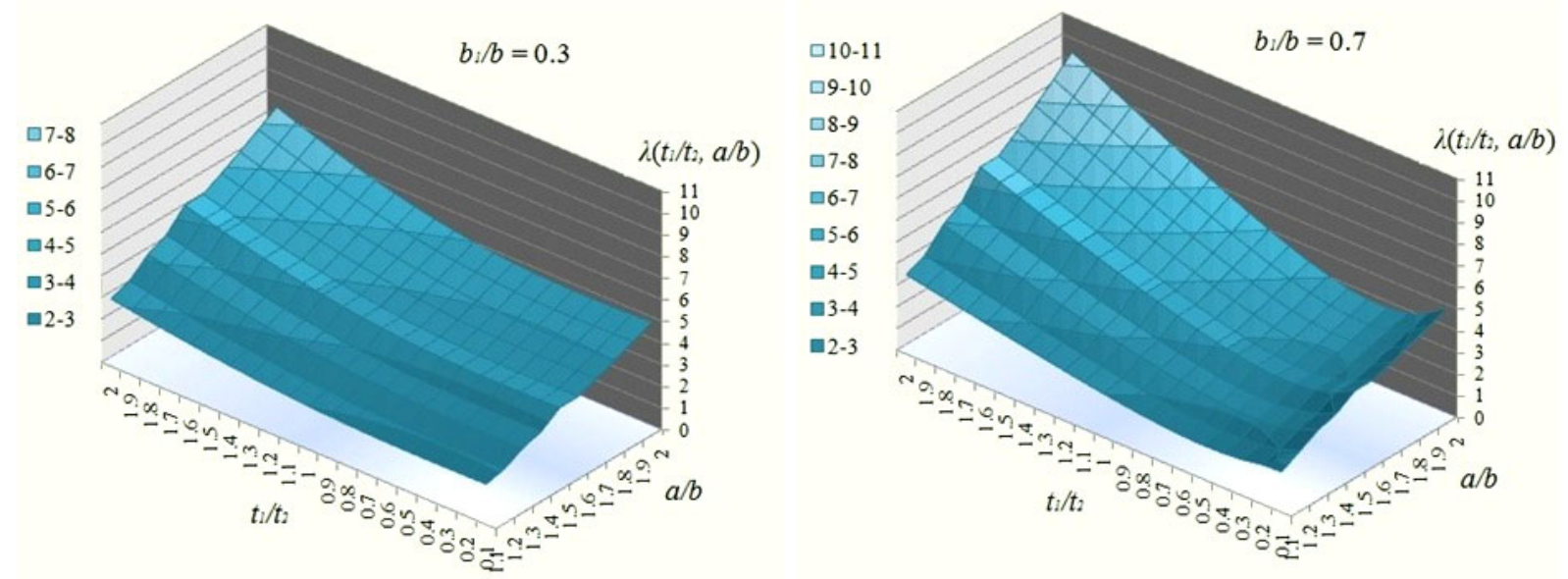

Fig. 4 Function of the frequency parameter $\lambda$ of the plate geometric dimensions for $m=1$

The minimum value of the function (24) corresponds to the value of $\Delta t=0.5$ for all values for the $a / b$ ratio. The three-dimensional function (24) is characterized by two changes along the $\Delta t$ direction for values of $a / b=1.3$ and 1.5, representing the phenomenon of "quasifrequency crossing". The occurrence of frequency crossing is characteristic of the higher modes of buckling when the reduction in $\lambda$ coefficient occurs between two modes of vibration. This phenomenon can partially happen in the first mode at the ratio $a / b=1.4$ (Fig. 4). The frequency parameter $\lambda$ for the second mode is characterized by the uniformity of distribution, whose minimum value obtained from (25) corresponds to the amount of $\Delta t=0.4$ for all the values of $a / b$ (Fig. 5). Increasing the dimension of $b_{1}$, i.e. reducing the relative plate width $\Delta b$, the frequency parameter $\lambda$ has a growing tendency for all modes of vibration. 

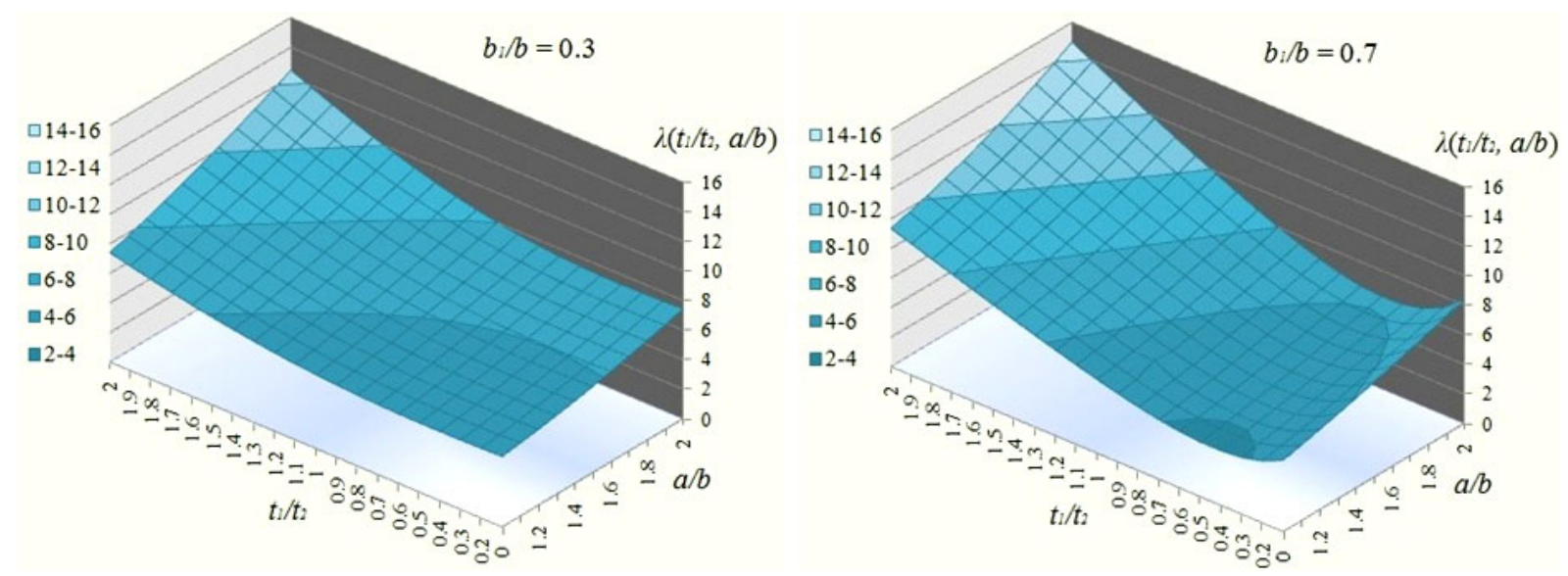

Fig. 5 Function of the frequency parameter $\lambda$ of the plate geometric dimensions for $m=2$

\section{Verification of the results}

The methodology applied and the results obtained were validated based on a comparative analysis using literature data for specific geometrical parameters obtained by using different methods (Table 2). Values of the buckling coefficient $k$ obtained in the present study are in quantitative and qualitative agreement with the reference values.

Table 2 Comparison of the buckling coefficient $k$ for the first mode

\begin{tabular}{|c|c|c|c|c|c|c|c|}
\hline & \multirow[b]{2}{*}{$m$} & \multirow[b]{2}{*}{$a / b$} & \multirow[b]{2}{*}{$t_{1} / t_{2}$} & \multicolumn{3}{|c|}{$b_{2} / b$} & \multirow{2}{*}{$\begin{array}{c}\text { mean } \\
\text { difference }[\%]\end{array}$} \\
\hline & & & & 0.3 & 0.5 & 0.7 & \\
\hline Present study & \multirow{4}{*}{1} & \multirow{4}{*}{1.0} & \multirow{4}{*}{1.2} & 6.094 & 5.272 & 4.650 & \\
\hline Xiang and Wei [9] & & & & 5.738 & 4.961 & 4.509 & 5.8 \\
\hline $\begin{array}{l}\text { Finite difference method } \\
{[14]}\end{array}$ & & & & 5.694 & 4.941 & 4.477 & 6.3 \\
\hline Xiang and Wang [3] & & & & 5.981 & 5.199 & 4.609 & 1.4 \\
\hline Present study & \multirow{2}{*}{1} & \multirow{2}{*}{1.0} & \multirow{2}{*}{1.5} & 10.615 & 7.553 & 5.762 & \\
\hline Xiang and Wang [3] & & & & 9.501 & 7.065 & 5.593 & 6.5 \\
\hline
\end{tabular}

Numerical data for the first mode of plate vibration which were obtained by using (24) are shown in Table 3 and Table 4 . As indicated by the comparative analysis of data obtained for the frequency parameter $\lambda$, the presented data is consistent with the data presented in studies $[3,21]$. It should be borne in mind that certain deviations were exhibited at larger plate discontinuities (higher relative thickness $t_{1} / t_{2}$ ). Results for the frequency parameter obtained in the study [21] display a clear tendency towards certain deviations at higher relative thickness of the plate. This fact is the result of approximation of the last two boundary conditions related to the continuity of bending moment and the transverse force along the connection line of the plates [21]. The approximation method has neglected the tangential stress components; at high discontinuities these have a significant effect of reducing the plate eigenfrequency.

The study dealing with exact buckling and vibration solutions for stepped rectangular plates [3] is based on the Levy function and the numerical identification of the frequency parameter based on the boundary conditions along the discontinuity line. Based on the analysis of functional dependency which corresponds to the first mode of plate vibration (24), it can be concluded that the frequency parameter $\lambda$ rises progressively with an increase in the plate dimensions ratio $a / b$, particularly in the case of larger discontinuities (Fig. 4). It is necessary to point out that all the examples referred to in the literature are given for fictitious load, Fig. $1 b$. 
Table 3 Comparison of the frequency parameter $\lambda$ for the first mode of vibration

\begin{tabular}{|c|c|c|c|c|c|c|c|c|c|}
\hline & \multirow[b]{2}{*}{$m$} & \multirow[b]{2}{*}{$a / b$} & \multirow[b]{2}{*}{$b_{1} / b$} & \multicolumn{6}{|c|}{$t_{1} / t_{2}$} \\
\hline & & & & 0.5 & 0.8 & 1.0 & 1.1 & 1.2 & 1.3 \\
\hline Present study & & & & 1.448 & 1.728 & 2.000 & 2.056 & 2.313 & 2.478 \\
\hline Chopra [21] & 1 & 1.0 & 0.75 & - & - & 2.000 & 2.199 & 2.437 & 2.724 \\
\hline Xiang [3] & & & & 1.293 & 1.703 & 2.000 & - & - & - \\
\hline Present study & & & & 0.874 & 1.062 & 1.250 & 1.352 & 1.458 & 1.568 \\
\hline Chopra [21] & 1 & 1.0 & 0.50 & - & - & 1.250 & 1.314 & 1.379 & 1.443 \\
\hline Xiang [3] & & & & 0.897 & 1.117 & 1.250 & - & - & - \\
\hline Present study & & & & 1.857 & 1.916 & 2.000 & 2.056 & 2.122 & 2.199 \\
\hline Chopra [21] & 1 & 1.0 & 0.25 & - & - & 2.000 & 2.018 & 2.033 & 2.047 \\
\hline Xiang [3] & & & & 1.629 & 1.889 & 2.000 & - & - & - \\
\hline
\end{tabular}

Table 4 Comparison of the frequency parameter $\lambda$ for the first and second modes of vibration

\begin{tabular}{|c|c|c|c|c|c|c|c|c|c|}
\hline & \multirow[b]{2}{*}{$m$} & \multirow[b]{2}{*}{$t_{1} / t_{2}$} & \multirow[b]{2}{*}{$b_{1} / b$} & \multicolumn{6}{|c|}{$a / b$} \\
\hline & & & & 0.50 & 0.75 & 1.00 & 1.25 & 1.50 & 2.00 \\
\hline Present study & \multirow{2}{*}{1} & \multirow{2}{*}{1.1} & \multirow{2}{*}{0.50} & 1.316 & 1.646 & 2.107 & 2.700 & 3.424 & 5.267 \\
\hline Chopra [21] & & & & 1.314 & - & 2.101 & - & - & 5.243 \\
\hline Present study & \multirow{2}{*}{1} & \multirow{2}{*}{1.2} & \multirow{2}{*}{0.50} & 1.391 & 1.739 & 2.227 & 2.853 & 3.619 & 5.567 \\
\hline Chopra [21] & & & & 1.379 & - & 2.202 & - & - & 5.463 \\
\hline Present study & \multirow{2}{*}{1} & \multirow{2}{*}{1.3} & \multirow{2}{*}{0.50} & 1.473 & 1.842 & .2357 & 3.021 & 3.831 & 5.894 \\
\hline Chopra [21] & & & & 1.443 & - & 2.299 & - & - & 5.651 \\
\hline Present study & \multirow{2}{*}{2} & \multirow{2}{*}{1.1} & \multirow{2}{*}{0.50} & 4.477 & 4.086 & 5.267 & 5.860 & 6.584 & 8.428 \\
\hline Chopra [21] & & & & - & - & 5.243 & - & - & 8.457 \\
\hline Present study & \multirow{2}{*}{2} & \multirow{2}{*}{1.2} & \multirow{2}{*}{0.50} & 4.732 & 5.080 & 5.567 & 6.194 & 6.959 & 8.908 \\
\hline Chopra [21] & & & & - & - & 5.463 & - & - & 9.049 \\
\hline Present study & \multirow{2}{*}{2} & \multirow{2}{*}{1.3} & \multirow{2}{*}{0.50} & 5.010 & 5.379 & 5.894 & 6.558 & 7.368 & 9.431 \\
\hline Chopra [21] & & & & - & - & 5.651 & - & - & 9.801 \\
\hline
\end{tabular}

\section{Conclusion}

The presented paper discusses the analytical method of determining the elastic buckling coefficient $k$ for the stepped plate subjected to a combined uniaxial load (defined as $\alpha$ ). The numerical data and diagrams of distribution of the parameter $k$ are given for two boundary cases $(\alpha=0$ and $\alpha=2)$ corresponding to uniform compression and pure bending. The application of stepwise variable plates exposed to constant pressure was deemed reasonable, and their use in the cases of linearly varying loads was necessary. The size of discontinuity as defined by relative thickness $\Delta t$ has the dominant influence on the buckling coefficient of the stepped plate. For the same geometric parameters of the plate, the load variant $\alpha=2$ is characterized by higher number buckling modes than it is the case when $\alpha=0$, in particular at higher discontinuities, which contributes to stability and facilitates the reduction in the critical buckling stress. The explicit formulation of frequency parameter $\lambda$ enables the geometry of plates subjected to dynamic loads to be optimized in order to achieve a minimum sensitivity to free vibration being induced. The specific contribution of this research is the significant data provided for the linearly varying load $(\alpha=2)$ of plates of stepwise variable thickness, which so far has failed to be the issue of systematic analyses in the literature on this subject matter. The influence of fictitious load, Fig. 1b, on the accuracy of the stability and natural vibration will be investigated by FEM (commercial package) in the future work. 


\section{Appendix A}

$$
\begin{aligned}
& \int_{-\frac{b}{2}}^{b_{1}-\frac{b}{2}} N_{x}(y) t_{1} y \mathrm{~d} y=\frac{1}{2} N_{1} t_{1}\left[(b / 2)^{3}-\left(b_{1}-b / 2\right)^{3}\right] \Rightarrow N_{1}=\frac{2}{3} \frac{b^{3}+\left(b_{1}-b / 2\right)^{3}}{b\left[(b / 2)^{3}-\left(b_{1}-b / 2\right)^{3}\right]} N_{o} \\
& \int_{b_{1}-\frac{b}{2}}^{\frac{b}{2}} N_{x}(y) t_{2} y \mathrm{~d} y=N_{2} t_{2} b_{1}\left(3 b_{1}-b\right) \Rightarrow N_{2}=\frac{2}{3} \frac{b^{3}-\left(b_{1}-b / 2\right)^{3}}{b_{1} b\left(3 b_{1}-b\right)} N_{o}
\end{aligned}
$$

\section{Appendix B}

$$
\begin{aligned}
& \delta_{m}=\varepsilon_{m}^{2}(1-k-\lambda) \\
& \varepsilon_{m}=(m \pi)^{2} \\
& \varphi=\frac{1}{a}
\end{aligned}
$$

\section{Appendix C}

$$
\begin{aligned}
& B_{1}=\left[\left(\frac{t_{1}}{t_{2}}\right)^{3} \phi+1-\phi\right]\left[1+\left(\frac{a}{b}\right)^{4}+2\left(\frac{a}{b}\right)^{2}\right]-\left[\left(\frac{t_{1}}{t_{3}}\right)^{3}-1\right]\left(\frac{\sin 2 \pi \phi}{2 \pi}\right)\left[1+\left(\frac{a}{b}\right)^{4}+2(2 v-1)\left(\frac{a}{b}\right)^{2}\right] \\
& B_{2}=\left[\left(\frac{t_{1}}{t_{2}}\right)^{3} \phi+1-\phi\right]\left[16+\left(\frac{a}{b}\right)^{4}+8\left(\frac{a}{b}\right)^{2}\right]-\left[\left(\frac{t_{1}}{t_{3}}\right)^{3}-1\right]\left(\frac{\sin 2 \pi \phi}{2 \pi}\right)\left[1+\left(\frac{a}{b}\right)^{4}+8(2 v-1)\left(\frac{a}{b}\right)^{2}\right] \\
& C=\left[\left(\frac{t_{1}}{t_{2}}\right) \phi+1-\phi\right]-\left[\left(\frac{t_{1}}{t_{3}}\right)-1\right]\left(\frac{\sin 2 \pi \phi}{2 \pi}\right) \\
& \phi=\frac{b_{1}}{b}
\end{aligned}
$$

\section{REFERENCES}

[1] Hosseini-Hashemi, S.; Khorshidi, K.; Amabili, M. Exact solution for linear buckling of rectangular Mindlin plates, Journal of Sound and Vibration 2008, 315(1), 318-342. https://doi.org/10.1016/j.jsv.2008.01.059

[2] Xiang, Y.; Zhao, Y.B.; Wei, G.W. Levy solutions for vibration of multi-span rectangular plates, International Journal of Mechanical Sciences 2002, 44(6), 1195-1218. https://doi.org/10.1016/S0020-7403(02)00027-9

[3] Xiang, Y.; Wang, C.M. Exact buckling and vibration solutions for stepped rectangular plates, Journal of Sound and Vibration 2002, 250(3), 503-517. https://doi.org/10.1006/jsvi.2001.3922

[4] Radosavljević, V.; Dražić, M. Exact solution for buckling of FCFC stepped rectangular plates, Applied Mathematical Modelling 2010, 34(12), 3841-3849. https://doi.org/10.1016/j.apm.2010.04.002

[5] Szilard, R. Theories and Applications of Plate Analysis, John Wiley \& Sons, 2004.

[6] Paik, J.K.; Thayamballi, A.K. Buckling strength of steel plating with elastically restrained edges, ThinWalled Structures 2000, 37(1), 27-55. https://doi.org/10.1016/S0263-8231(00)00009-4

[7] Gorman, D.J. Free vibration and buckling of in-plane loaded plates with rotational elastic edge support, Journal of Sound and Vibration 2000, 229(4), 755-773. https://doi.org/10.1006/jsvi.1999.2527

[8] Zhang, X.; Li, W.L. Vibrations of rectangular plates with arbitrary non-uniform elastic edge restraints, Journal of Sound and Vibration 2009, 326(1), 221-234. https://doi.org/10.1016/j.jsv.2009.04.021

[9] Timoshenko, S.P.; Gere, J.P. Theory of elastic stability, $2^{\text {nd }}$ Ed. New York: McGraw-Hill Book Company 1961. 
[10] Xiang, Y.; Wei, G.W. Exact solutions for buckling and vibration of stepped rectangular Mindlin plates, International Journal of Solids and Structures 2004, 41(1), 279-294. https://doi.org/10.1016/j.ijsolstr.2003.09.007

[11] Leissa, A.W.; Kang, J.H. Exact solutions for vibration and buckling of an SS-C-SS-C rectangular plate loaded by linearly varying in-plane stresses, International Journal of Mechanical Sciences 2002, 44(9), 1925-1945. https://doi.org/10.1016/S0020-7403(02)00069-3

[12] Wang, X.; Gan, L.; Wang, Y. A differential quadrature analysis of vibration and buckling of an SS-C-SS$\mathrm{C}$ rectangular plate loaded by linearly varying in-plane stresses, Journal of Sound and Vibration 2006, 298(1), 420-431. https://doi.org/10.1016/j.jsv.2006.06.003

[13] Madhavan, M.; Davidson, J.S. Buckling of centerline-stiffened plates subjected to uniaxial eccentric compression, Thin-Walled Structures 2005, 43(8), 1264-1276. https://doi.org/10.1016/j.tws.2005.03.013

[14] Jaberzadeh, E.; Azhari, M. Elastic and inelastic local buckling of stiffened plates subjected to nonuniform compression using the Galerkin method, Applied Mathematical Modelling 2009, 33(4), 1874-1885. https://doi.org/10.1016/j.apm.2008.03.020

[15] Wilson, A.J.; Rajasekaran, S. Elastic stability of all edges simply supported, stepped and stiffened rectangular plate under biaxial loading, Applied Mathematical Modelling 2014, 38(2), 479-495. https://doi.org/10.1016/j.apm.2013.06.028

[16] Fan, S.C.; Cheung, Y.K. Flexural free vibrations of rectangular plates with complex support conditions, Journal of Sound and Vibration 1984, 93(1), 81-94. https://doi.org/10.1016/0022-460X(84)90352-3

[17] Cheung, Y.K. Finite Strip Method in Structural Analysis, Pergamon Press, Oxford, UK, 1976.

[18] Wang, X.; Wang, Y.; Wu, Z. Differential quadrature analysis of free vibration of rhombic plates with free edges, Applied Mathematics and Computation 2013, 225, 171-183. https://doi.org/10.1016/j.amc.2013.09.018

[19] Rahai, A.R.; Alinia, M.M.; Kazemi, S. Buckling analysis of stepped plates using modified buckling mode shapes, Thin-Walled Structures 2008, 46(5), 484-493. https://doi.org/10.1016/j.tws.2007.10.012

[20] Bodaghi, M.; Saidi, A.R. Levy-type solution for buckling analysis of thick functionally graded rectangular plates based on the higher-order shear deformation plate theory, Applied Mathematical Modelling 2010, 34(11), 3659-3673. https://doi.org/10.1016/j.apm.2010.03.016

[21] Harik, E.I.; Balakrishnan, N. Stability of rectangular orthotropic plates, Applied MathematicalMechanics 1994, 18(7), 400-402. https://doi.org/10.1016/0307-904X(94)90226-7

[22] Chopra, I. Vibration of stepped thickness plates, International Journal of Mechanical Sciences 1974, 16(6), 337-344. https://doi.org/10.1016/0020-7403(74)90007-1

[23] Cho, D.S., Kim, B.H., Kim, J.H., Vladimir, N., Choi, T.M. Simplified dynamic analysis of stepped thickness rectangular plate structures by the assumed mode method, Proceedings of the Institution of Mechanical Engineers, Part M: Journal of Engineering for the Maritime Environment 2017, 231(1), 177187. https://doi.org/10.1177\%2F1475090216630001

[24] Cho, D.S., Choi, T.M., Kim, J.H., Vladimir, N. Structural intensity analysis of stepped thickness rectangular plates utilizing the finite element method, Thin-Walled Structures 2016, 109, 1-12. https://doi.org/10.1016/j.tws.2016.09.015

[25] Senjanović, I., Vladimir, N., Hadžić, N. Modified Mindlin plate theory and shear locking-free finite element formulation, Mechanics Research Communications 2014, 55, 95-104. https://doi.org/10.1016/j.mechrescom.2013.10.007

[26] Senjanović, I., Vladimir, N., Cho, D.S. A new finite element formulation for vibration analysis of thick plates, International Journal of Naval Architecture and Ocean Engineering 2015, 7(2), 324-345. https://doi.org/10.1515/ijnaoe-2015-0023

[27] Đelošević, M.; Vukajlov, Lj.; Tepić, G.; Milinković, A.; Brkljač, D. Design of Columns in Terms of Stability, Transactions of FAMENA 2018, 42(2), 39-50. https://doi.org/10.21278/TOF.42204

[28] Braun, M. Differential equations and their applications. $4^{\text {th }}$ Ed. New York: Springer 1993.

Submitted: $\quad 15.01 .2020$

Accepted: $\quad 28.9 .2020$
Mirko Đelošević

djelosevic.m@uns.ac.rs

Goran Tepić*

gtepic@uns.ac.rs

University of Novi Sad, Faculty of

Technical Sciences, Trg Dositeja

Obradovića 6, Novi Sad, Serbia 